\title{
Investigating the genomic alteration improved the clinical outcome of aged patients with lung carcinoma
}

\author{
Sixian Chen ${ }^{1 \dagger}$, Aizhen Fu ${ }^{2 \dagger}$, Yuan Lu', Wei Lu', Yongfeng Chen' ${ }^{1}$, Shuiqiang Hong', Suli Zhou', Tianmin Xiang ${ }^{3}$, \\ Zhenzhen Zhang ${ }^{3^{*}}$ and Yongguang Cai ${ }^{{ }^{*}}$
}

\begin{abstract}
Background: Lung carcinoma is a common geriatric disease. The development of genotype-targeted therapies greatly improved the management of lung carcinoma. However, the treatment for old patients can be more complex than that for young individuals.

Results: To investigate the benefits of genetic detection for older patients with lung carcinoma, we explored the genomic profiling of 258 patients with more than 55 years using a targeted next generation sequencing, and some of these patients were treated with targeted therapies based on the results of genomic detection. KRAS codon 61 mutations were found in 15.2\% KRAS-mutated patients, which tend to be co-existing with other classical activating mutations other than codons 12/13. Acquired EGFR C797S mutations were identified in 2 cases and ERBB2 amplification was identified in 1 case. All these 3 cases developed resistance to EGFR tyrosine kinase inhibitors and showed expected results of their followed therapies. The median progression-free survival and median overall survival of patients treated with molecular targeted therapies were better than those of patients treated with chemoradiotherapy alone.
\end{abstract}

Conclusions: Our findings revealed the specific genomic profiles of patients older than 55 years with lung carcinoma and suggested that these old patients have been benefit from the genetic detection, which helped identify druggable mutations and distinguish resistance mechanisms.

Keywords: Lung cancer, Genomic alterations, KRAS codon 61, Targeted treatment, Mechanism of resistance

\section{Background}

Lung cancer, one of the most common malignancies worldwide, is the leading cause of cancer mortality [1]. The incidence of lung cancer is relatively low (1-10\%) in the young adult patients [2], and increases with age [3]. Due to the aging trend observed in societies and

\footnotetext{
*Correspondence: zhenzhen.zhang@singlera.com; caiyongguang@126.com

${ }^{\dagger}$ Sixian Chen and Aizhen Fu contributed equally to this work.

${ }^{1}$ Medical Oncology Department V, Guangdong Nongken Central

Hospital, Zhanjiang 524002, China

${ }^{3}$ Singlera Genomics Inc., Shanghai 201318, China

Full list of author information is available at the end of the article
}

the increasing availability of high-resolution computed tomography (CT) and positron emission tomography (PET), the incidence of lung cancer in older patients is markedly increasing.

The treatment of older patients with lung cancer can be more complex than that of younger patients due to the age-related decline in organ function, multiple comorbidities, concurrent medications, and possible presence of a geriatric syndrome $[4,5]$, which have the potential to increase drug-related toxicity and affect the ability of older patients to tolerate and continue treatment. The challenge for clinicians is how to select a treatment 
strategy for older patients that prolongation of survival time while maintaining quality of life.

Despite studies have showed that older patients with minimal comorbid conditions tolerated and respond to chemo just as well as young patients, older patients are not prescribed standard chemotherapy [6]. In recent years, many lung cancer patients have benefited from the personalized treatment like targeted therapy on the basis of the genetic background of the tumor [7]. EGFR mutations, $A L K$ rearrangements and ROS1 rearrangements are regarded as "must test" biomarkers in the molecular diagnosis of advanced lung carcinoma patients [8]. For the development of genotype-targeted therapies, it is necessary to identity oncogenic molecular changes leading to aberrant activation of intracellular signaling associated with the sustained growth of lung cancer cells. Other oncogenic alterations have been revealed like $M E T, B R A F$ and $H E R 2$ as novel targets for personalized therapies $[9,10]$ by the next-generation sequencing (NGS) technology. Considering the high incidence of lung cancer in the older population, comprehensive information regarding specific molecular abnormalities is valuable in choosing feasible treatment options to maximize therapeutic benefits and minimize therapy-associated risks.

In the present study, a well-validated 12 gene panel for genomic analysis was applied to 258 Chinese lung carcinoma patients with more than 55 years old. Their genomic alterations were used to help designing the following medical treatment. The main objective of this study was to investigate the molecular characteristics of these old patients with lung carcinoma in China, and to find out whether these old patients were benefited from the genetic detection.

\section{Results}

\section{Patient characteristics}

The clinical features of all 258 Chinese patients with lung carcinoma in the cohort are summarized in Table 1 with details in Additional file 1: Table S1. The median age of patients at the time of first sampling was 69 years (range 55 to 99 years). Among these patients, 202 (78.3\%) were classified as stage IV, and 44 (17.1\%) were as stage I, II and III. For all the 258 patients enrolled, 249 were sampled once, while other 9 patients were sampled both before and after EGFR tyrosine kinase inhibitors (TKIs) treatment (Table 2).

\section{Molecular profiling of lung carcinoma in aged patients}

Mutation analysis revealed that 217 samples harbored at least one gene alteration (Fig. 1). As shown in Fig. 1, the most frequently mutated genes TP53 (46.4\%, 124/267) and EGFR $(44.2 \%, 118 / 267)$ were identified in about
Table 1 The baseline characteristics of 258 patients with lung carcinoma

\begin{tabular}{lll}
\hline Variables & $\mathbf{n = 2 5 8}$ patients & \% \\
\hline Age (year) & & \\
Median (range) & 69 (55-99) & \\
Gender & & \\
$\quad$ Male & 135 & $52.3 \%$ \\
$\quad$ Female & 123 & $47.7 \%$ \\
Smoking status & & \\
Smokers & 90 & $34.9 \%$ \\
Never smoked & 160 & $62.0 \%$ \\
$\quad$ Unknown & 8 & $3.1 \%$ \\
Histologic types & & \\
Adenocarcinoma (ADC) & 172 & $66.7 \%$ \\
Squamous cell carcinoma (SCC) & 20 & $7.8 \%$ \\
Adenosquamous carcinoma (ASC) & 3 & $1.2 \%$ \\
Large cell carcinoma & 1 & $0.4 \%$ \\
Small-cell carcinoma & 3 & $1.2 \%$ \\
Unknown & 59 & $22.9 \%$ \\
Clinical stage & & \\
I & 5 & $1.9 \%$ \\
II & 4 & $1.6 \%$ \\
III & 35 & $13.6 \%$ \\
IV & 202 & $78.3 \%$ \\
Unknown & 12 & $4.7 \%$ \\
\hline
\end{tabular}

Table 2 The number of patients from which samples were collected

\begin{tabular}{llll}
\hline & \multicolumn{3}{l}{ No. of patients } \\
\cline { 2 - 4 } & FFPE & Plasma & Both \\
\hline Sampled before treatment & 103 & 103 & - \\
Sampled after treatment & 8 & 35 & - \\
Targeted therapy & 7 & 22 & - \\
Targeted plus Chemotherapy & - & 3 & - \\
Chemotherapy & 1 & 10 & - \\
Sampled both before and after treatment & 2 & 4 & $3^{\text {a }}$ \\
\hline
\end{tabular}

${ }^{a}$ For the 3 patients, formalin-fixed paraffin-embedded (FFPE) samples were collected before treatment and plasma samples were collected after treatment

half of our cohort. KRAS mutations were found in 33 $(12.4 \%, 33 / 267)$ patients. Other genes were mutated in no more than $10 \%$ of the patients enrolled. Compared with the TCGA population (https://portal.gdc.cancer. gov), we found a more mutation frequency of EGFR (53.9\% vs. $14.6 \%, P<0.001$ ) and a less frequency of $K R A S$ $(15.2 \%$ vs. $27.9 \% ; P<0.001)$ in our cohort. Besides, EGFR mutation was more frequently observed in plasma samples compared to FFPE tissue specimens $(P=0.001)$. The most discrimination observed was the number of 


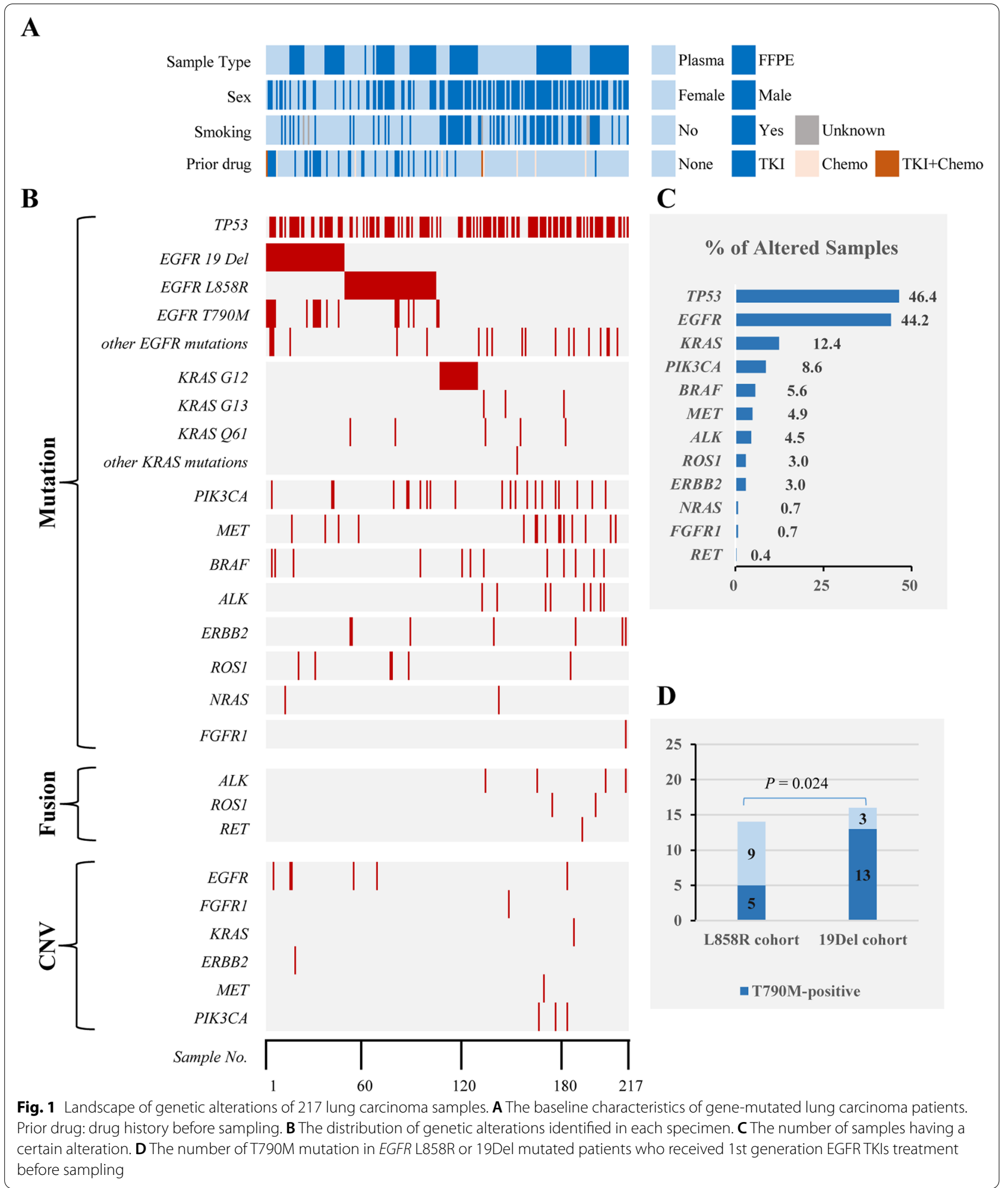

EGFR T790M (3 in FFPE and 18 in plasma samples). When we excluded those samples collected after medical treatment, no statistical difference of gene alteration was observed between the FFPE and plasma samples (Fig. 2). In addition, no difference was observed in specific mutational signature of single nucleotide variants 


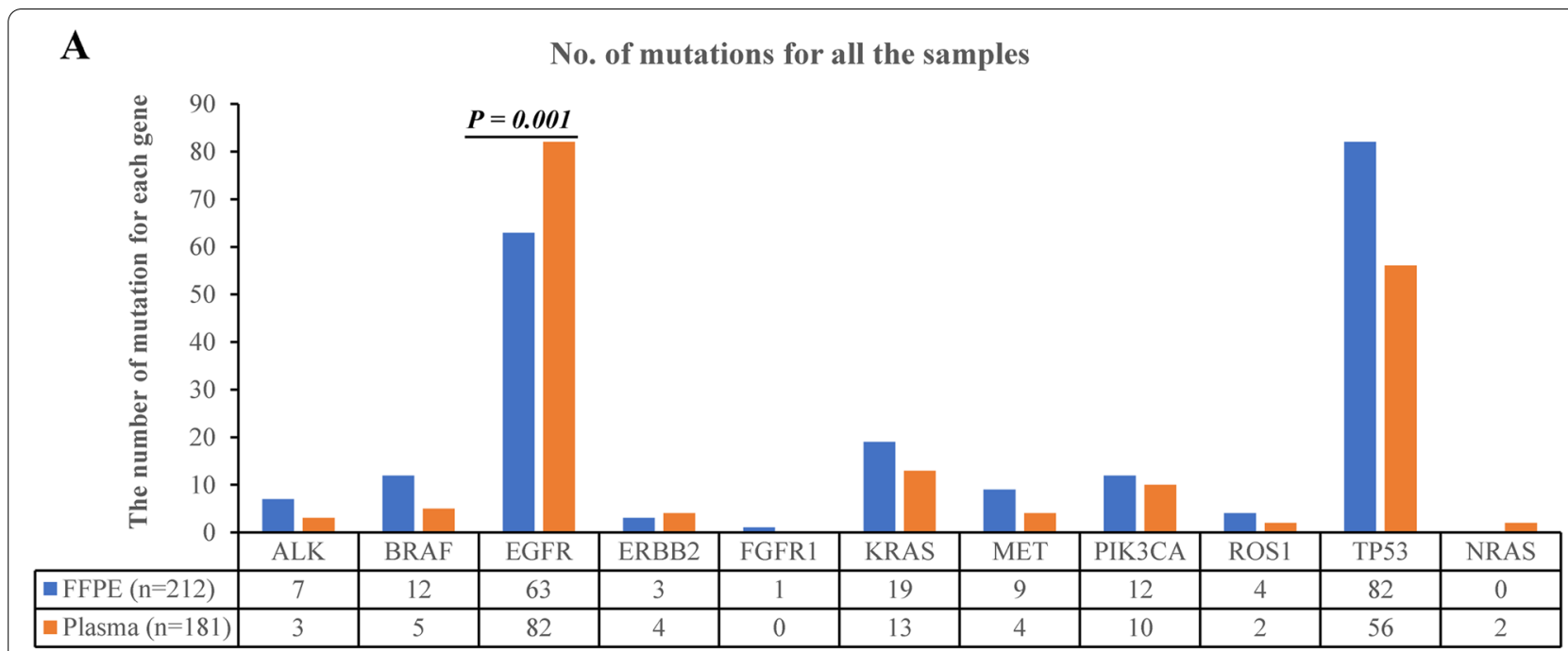

B

No. of mutations for the samples collected before medical treatment

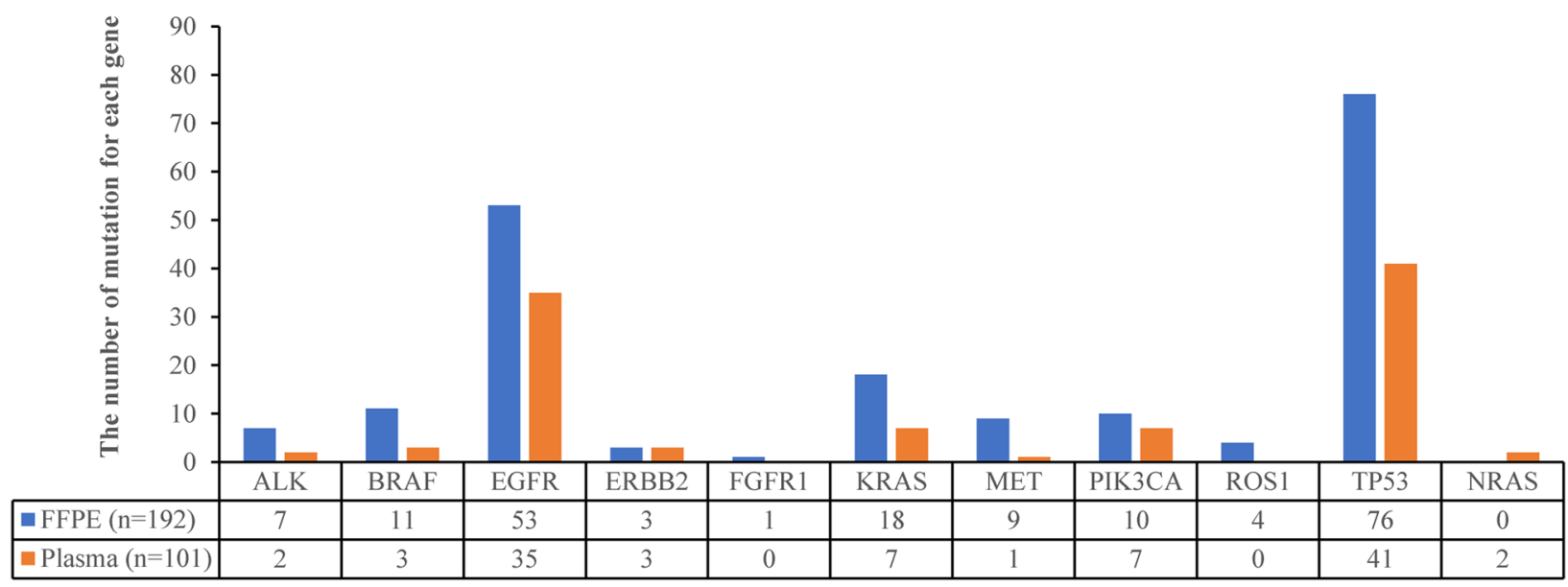

Fig. 2 Distribution of gene mutations in FFPE tissue and plasma

(SNVs) between the FFPE and plasma samples for either all patients enrolled or therapy- naïve patients (Additional file 2: Fig. S1).

\section{EGFR mutations}

EGFR exon 19 deletions $(47 / 267,17.6 \%)$ and exon 21 p.L858R mutation $(55 / 267,20.6 \%)$ were the most common mutations in EGFR gene. Ten EGFR 19del subtypes were confirmed in 47 samples from 43 patients (Table 3). Deletions starting from E746 occurred in 38 samples (80.9\%) and starting from L747 occurred in 9 ones (19.1\%). DelE746_A750 was the most frequent subtype (33/47, 70.2\%). For the patients receiving EGFR-TKIs treatment, no difference was found between patients with delE746_A750 and with other deletions in the objective response rate (ORR) ( $85 \%$ vs. $66.7 \%, P=0.558)$ and no difference between patients with deletions starting from E746 and from L747 in ORR (81.8 vs. 75\%, $P>0.999$; Table 3, Additional file 1: Table S2). In 30 EGFR-mutated patients who received 1st generation EGFR TKIs treatment, 5 harbored T790M in 14 individuals with L858R, while 13 carried T790M in 16 individuals with 19Del (Fig. 1D). Patients with EGFR 19Del were more likely to acquire T790M mutation than those with L858R after they were treated with EGFR TKIs $(P=0.024)$.

The EGFR mutations were more frequent in females than males $(56.1 \%$ vs. $29.6 \%, P<0.001$; Fig. $3 \mathrm{~A})$, in patients without smoking history than smokers $(55 \%$ vs. $20 \%, P<0.001$; Fig. $3 \mathrm{~B})$, in ADC patients than SCC patients $(51.7 \%$ vs. $25 \%, P<0.001$; Fig. $3 C)$, and in IV stage patients than I-III stage patients $(47.5 \%$ vs. $27.3 \%$, $P=0.014 ;$ Fig. 3D). 
Table 3 EGFR exon 19del subtypes in patients

\begin{tabular}{|c|c|c|c|c|}
\hline \multirow[t]{2}{*}{ No. } & \multirow[t]{2}{*}{ Subtypes } & \multirow{2}{*}{$\begin{array}{l}\text { Number } \\
\text { of altered } \\
\text { samples }\end{array}$} & \multicolumn{2}{|c|}{$\begin{array}{l}\text { TKI response } \\
\left(_{\text {total }=26)^{\mathrm{a}}}\right.\end{array}$} \\
\hline & & & PR, N & PD, $N$ \\
\hline \multirow[t]{2}{*}{1} & c.2235_2249del & & & \\
\hline & p.Glu746_Ala750del & 20 & 10 & 2 \\
\hline \multirow[t]{2}{*}{2} & c.2236_2250del & & & \\
\hline & p.Glu746_Ala750del & 13 & 7 & 1 \\
\hline \multirow[t]{2}{*}{3} & c.2236_2248delinsAGCC & & & \\
\hline & p.Glu746_Ala750delinsSerPro & 1 & & 1 \\
\hline \multirow[t]{2}{*}{4} & c.2236_2252delinsCT & & & \\
\hline & p.Glu746_Thr751delinsLeu & 1 & & \\
\hline \multirow[t]{2}{*}{5} & c.2236_2257delinsCTCT & & & \\
\hline & p.Glu746_Pro753delinsLeuSer & 1 & & \\
\hline \multirow[t]{2}{*}{6} & c.2237_2252delinsT & & & \\
\hline & p.Glu746_Thr751delinsVal & 1 & & \\
\hline \multirow[t]{2}{*}{7} & c.2237_2255delinsT & & & \\
\hline & p.Glu746_Ser752delinsVal & 1 & 1 & \\
\hline \multirow[t]{2}{*}{8} & c.2239_2247del & & & \\
\hline & p.Leu747_Glu749del & 1 & & \\
\hline \multirow[t]{2}{*}{9} & c.2239_2256del & & & \\
\hline & p.Leu747_Ser752del & 1 & & \\
\hline \multirow[t]{2}{*}{10} & c.2240_2254del & & & \\
\hline & p.Leu747_Thr751del & 2 & 1 & 1 \\
\hline \multirow[t]{2}{*}{11} & c.2240_2257del & & & \\
\hline & p.Leu747_Pro753delinsSer & 5 & 2 & \\
\hline
\end{tabular}

a patients received the first-generation TKI treatment

\section{KRAS mutations}

KRAS mutations were observed in 33 samples, most of which were in codons $12 / 13(78.9 \%, 26 / 33)$ and codon $61(15.2 \%, 5 / 33)$. Compared to codons $12 / 13$, codon 61 mutations more tend to overlap with other frequent oncogenic alterations like EGFR L858R, 19Del, $A L K$ rearrangement and BRAF V600E (60\% vs. $3.85 \%, P=0.008)$. No patient with mutations in codons $12 / 13$ received targeted therapy, whereas three patients with KRAS codon 61 mutations showed stable disease or partial response after receiving TKI inhibitor ((Additional file 1: Table S3).

KRAS mutation status was found have association with smoking history $(21.1 \%$ for smokers vs. $8.8 \%$ for nonsmokers, $P=0.006$; Fig. $3 \mathrm{E})$, and gender $(17.8 \%$ for males vs. $7.3 \%$ for females, $P=0.012$; Fig. $3 \mathrm{~F}$ ). No association was found between KRAS mutation status and the histologic types, and clinical stage (Additional file 1: Table S4).

\section{TP53 mutations}

A total of 110 mutation types were identified in our cohort, most of which were observed in only one sample (Additional file 1: Table S1). The majority of these mutations were located within the p53 DNA-binding domains (exons $5-8$ ), including $\mathrm{R} 175 \mathrm{H}$ in 4 samples, R273L in 3 samples, etc.

The TP53 mutation frequency in males was much higher than that in females ( $56.3 \%$ for vs. $35.0 \%, P<0.001$; Fig. 3G). The TP53 mutated more frequency in patients with tobacco consumption than that in patients without smoking history ( $57.8 \%$ for vs. $40.6 \%, P=0.009$; Fig. $3 \mathrm{H}$ ). The SCC had a higher frequency of TP53 mutations compared to ADC individuals $(80.0 \%$ vs. $47.1 \%, P=0.005$; Fig. 3I). No difference was found in the clinical stage between the TP53 mutation (Additional file 1: Table S4).

Among the 107 samples with multiple gene mutations, concomitant EGFR and TP53 mutations were found in 63 ones (58.9\%). According to the follow-up data collected, only $34 E G F R$-mutated patients were included in survival analysis. The median PFS was 8.6 months for TP53 mutated patients and 9.8 months for TP53 wildtype patients. No difference in PFS was found between EGFR-mutated patients with and without TP53 mutations $(P=0.491$; Fig. 3J).

\section{Genomic detection could improve clinical outcomes}

In the current study, we described three patients (P6, P7, P8) with multiple gene detection results during the whole treatment history to see if the genomic characterization helped their clinical management (Fig. 4). The three patients all had acquired resistance to EGFR TKIs.

The first patient (P6) was a 63-year-old man diagnosed with lung adenocarcinoma with metastases to bone in July 2016. Because of the 19Del of EGFR identified by Amplification Refractory Mutation System (ARMS), he was treated with gefitinib and achieved partial responses (PR) for 11 months. However, progression disease (PD) was observed, and EGFR 19Del and acquired T790M were both identified by NGS in plasma in May 2017. Osimertinib was added and the patient responded for another 11 months. An acquired cis-C797S, which lead the drug resistant, besides with 19Del and T790M mutations were identified by NGS in plasma in June 2018. The patient was then treated with the combination of osimertinib and bevacizumab (4weeks), under which the disease did not stop progressing (Fig. 4).

The second patient (P7) was a 67-year-old woman diagnosed with lung adenocarcinoma with metastases to multiple organs in June 2016. Due to the presence of 19Del by ARMS, she was treated with gefitinib, which resulted in clinically PR. About 11 months after therapy, CT scan showed increased lesions in both liver and lung. After 2 cycles of chemotherapy, the patient was treated with osimertinib and maintained PR for 11 months until December 2018. Then, EGFR cis-C797S combined with T790M and 19Del were 
A

Sex vs EGFR

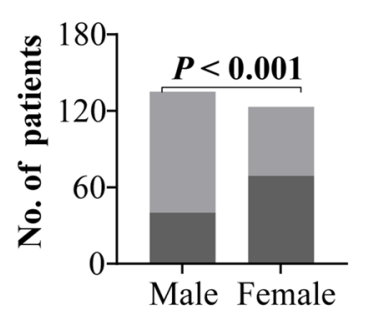

D

TNM stage vs $E G F R$

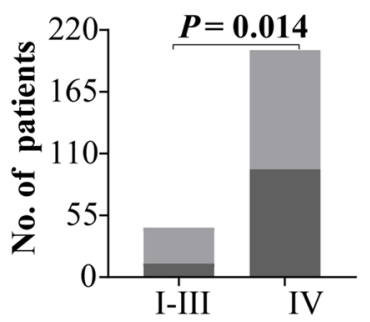

G

Sex vs TP53

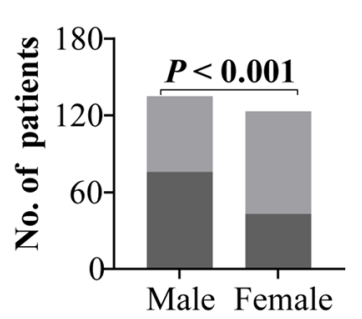

B

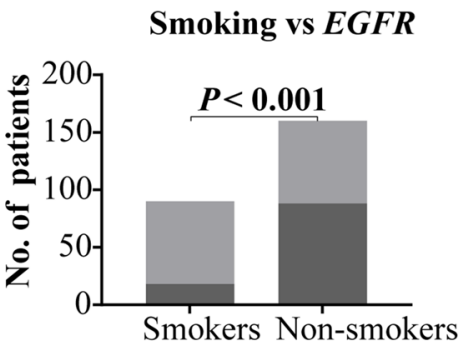

E

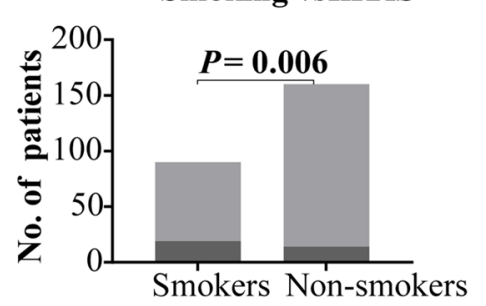

H
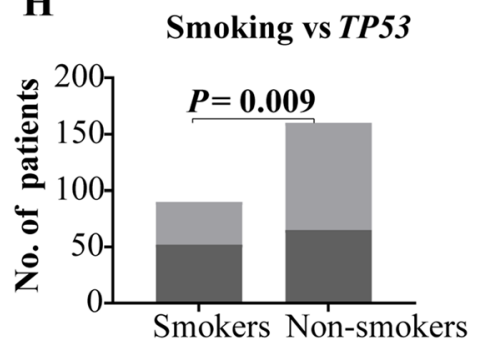

C

Histologic types vs EGFR

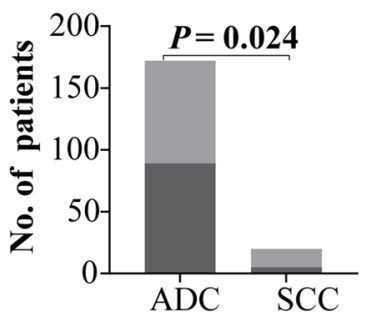

F

Sex vs KRAS

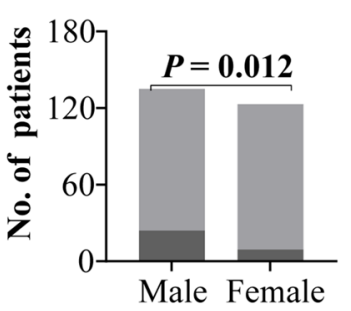

I

Histologic types vs TP53

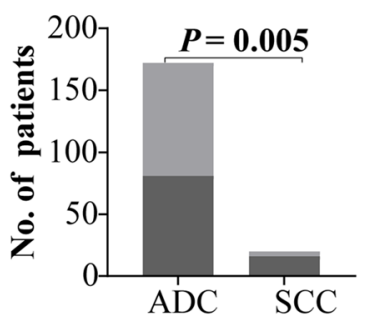

J

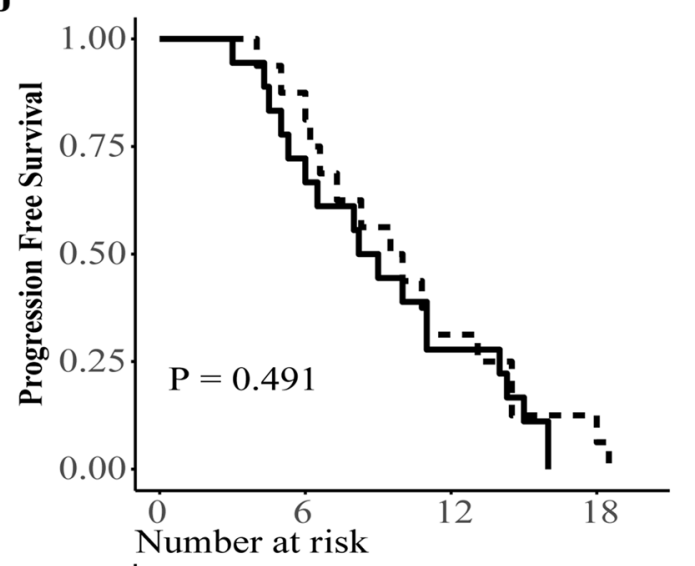

EGFR/KRAS/TP53-wild

EGFR/KRAS/TP53-mutated

- TP53-mutated

- - TP53-wild

ber at risk

\begin{tabular}{r|cccc} 
TP53-mutated & 18 & 13 & 5 & 0 \\
TP53-wild & 16 & 14 & 5 & 2 \\
\cline { 2 - 5 } & 0 & 6 & 12 & 18
\end{tabular}

Fig. 3 Analysis of mutations in EGFR, KRAS and TP53. A-I Correlation of patient clinical characteristics and the genetic alterations. J Progression free survival in 34 EGFR mutated patients with or without TP53 mutation. "Number at risk" is the total number of participants in the study exposed to the risk of outcome events 


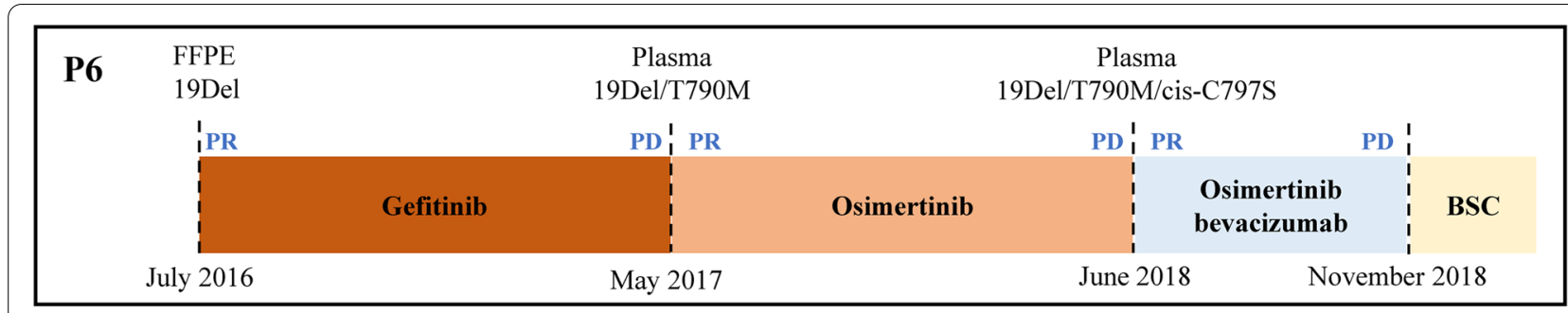
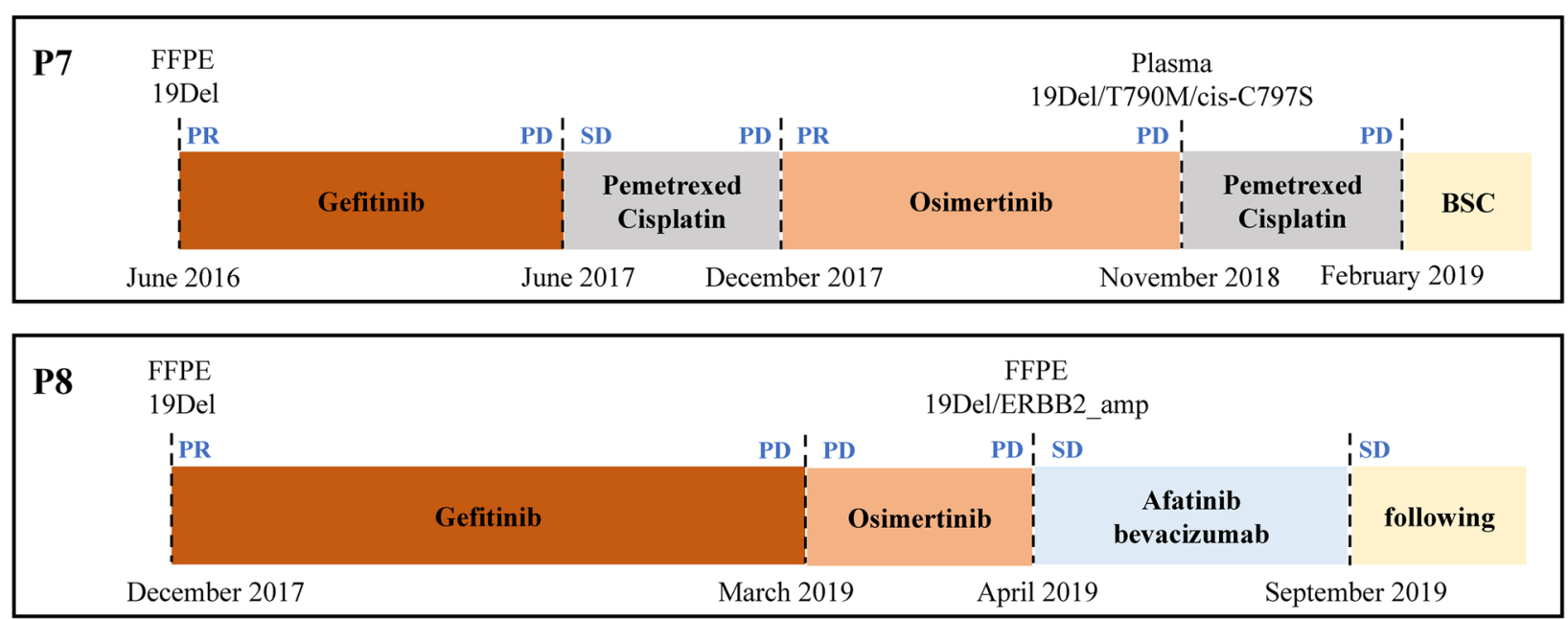

Fig. 4 The treatment history and driver gene evolution for 3 patients who had acquired resistance to EGFRTKIs. BSC, best supportive care

identified by NGS in plasma DNA. This patient was treated with a standard chemo strategy with pemetrexed-cisplatin, which had a poor effect (Fig. 4).

The third patient (P8) was a 56-year-old woman diagnosed with lung adenocarcinoma with metastases to bone. ARMS revealed EGFR 19Del mutation in patient's surgical specimen. She was treated with gefitinib and achieved PR for 16 months until PD. Without any genetic test, this patient was treated with Osimertinib, but no response was observed. Later, the NGS resulted from plasma DNA revealed an acquired $E R B B 2$ amplification in conjunction with 19Del. The patient was then treated with a combination of afatinib, bevacizumab, and radiotherapy, which resulted in clinically stable disease (SD) at the last follow-up on Sep 1, 2019 (Fig. 4).

\section{Association of treatment selections with patient survival}

The median PFS and median OS of patients treated with EGFR TKIs alone was 10.9 and 17.1 months, which showed significantly longer than those of patients (PFS: 5.0; OS: 8.0 months) treated with chemoradiotherapy $(P<0.001$ and $P=0.003$, respectively) (Fig. 5).

\section{Discussion}

Most lung cancer patients have no obvious specific symptoms at the beginning of the disease [11]. Older patients are usually with the high prevalence of comorbid disease that makes lung cancer more difficult to be detected at an early stage. In this study, lung cancer patients older than 55 years were enrolled, the majority of which were in stage IV. Comprehensive genomic spectrum on 267 lung cancer specimens from 258 patients aged more than 55 years showed a heterogeneity in the current study. The rare MET 14 exon mutations, as well as some common druggable genes like EGFR, KRAS, and $A L K$ were detected in cases that sampled before any treatment. $E R B B 2$ amplification, a bypass mechanism of resistance to EGFR TKIs, besides with the common EGFR T790M resistance mutation were identified in cases that sampled after EGFR TKIs treatment. We explored the genetic status of patients with both the FFPE and plasma samples, and no difference of gene alteration was found between the FFPE and plasma samples except for EGFR. The $E G F R$ mutation was also more frequent in stage IV compared to stage I-III patients. For these two situations, EGFR T790M which occurred during the tumor progression and medical treatment may be the major cause. Some samples for stage IV patients and some plasma 

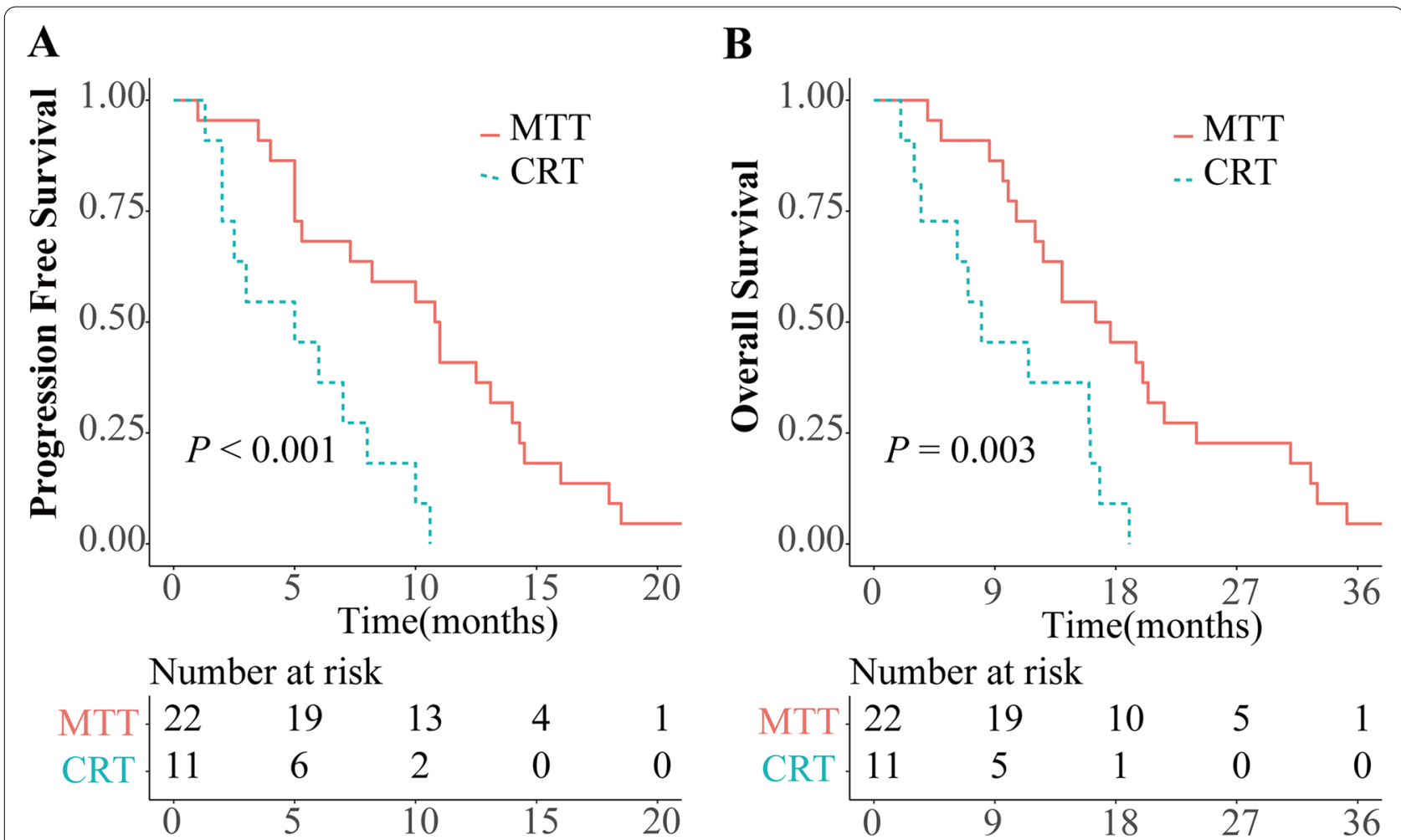

Number at risk

\begin{tabular}{|c|c|c|c|c|}
\hline MTT & 22 & 19 & 13 & 4 \\
\hline CRT & 11 & 6 & 2 & 0 \\
\hline & $\dot{0}$ & 5 & 10 & 15 \\
\hline
\end{tabular}

MTT $\left\{\begin{array}{ccccc}22 & 19 & 10 & 5 & 1 \\ 11 & 5 & 1 & 0 & 0 \\ \hline 0 & 9 & 18 & 27 & 36\end{array}\right.$

Fig. 5 Kaplan-Meier curves for progression-free survival (PFS) (A) and overall survival (OS) (B) of all 33 patients. MTT, molecular targeted therapy; CRT, chemoradiotherapy

samples were collected after a period of treatment, which resulted an increase of EGFR T790M. When we compare the samples collected before treatment, no difference of gene alteration was found between the FFPE and plasma samples. Similar to the reported [12], EGFR mutations were more likely to be occurred in non-smokers, female and ADC patients. Besides, we found that EGFR was mutated at a much higher frequency in our cohort than in Caucasians when we compared our data to the TCGA dataset. Studies showed that female sex, adenocarcinoma histology, never-smoking status, and Asian ethnicity are considered the most important factors associated with EGFR mutation [13], and mutations of KRAS and TP53 were more frequent in males and smokers other than females and non-smokers $[14,15]$. These biases were all validated in our results, which suggested that the characteristics of EGFR, KRAS and TP53 in our study were similar with others cohort.

$E G F R$ is a transmembrane tyrosine kinase receptor which activates Jak, PI3K, ROS, and RAS pathways leading to cell survival [16]. The presence of the main activating mutations, including L858R mutation and the exon 19Del is associated with sensitivity to EGFR-TKIs [17]. Studies showed that EGFR 19Del was associated with better outcomes in treatments with EGFR-TKIs than L858R mutation [18]. Some investigators have explored the structures of 19Del and L858R and suggested different binding affinity of TKIs [19]. Here, we reported a higher prevalence of T790M mutation in 19Del cohort than that in L858R cohort, consistent with previous research [20], this may be a mechanism of different outcomes of these two alteration subtypes. It was reported that the 19Del subtypes could also influence different clinical outcomes to EGFR-TKIs [21], while others found no significant differences between patients with different subtypes [22]. In this study, no significant differences in ORR between different EGFR 19Del subtypes. However, due to limited sample size, it was not reasonable to definitively conclude which subtype had the most important influence. The T790M mutation in the ATP-binding site of EGFR is the most common mechanism of resistance to 1st and 2nd generation EGFR TKIs [23]. The role of methionine (M) mutated from threonine ( $\mathrm{T}$ ) acts as a "gatekeeper" residue causing steric hindrance thus decreasing hydrophilicity and preventing tyrosine kinase binding [24]. Moreover, T790M mutation increases the affinity for ATP in EGFR kinase causes drug resistance [25]. Other mechanism for resistance like EGFR C797S mutation [26], and ERBB2 amplification [23], were also identified in our studies. We presented the clinical procedure of 3 
subjects and demonstrated the value of genomic detection for helping clinicians to distinguish specific resistance mechanisms in each patient and make personalized medicine strategies. Genomic evolution of tumor is the major obstacle in long-term response to TKI treatment. So that it is necessary for lung cancer patients, especially the old patients with high heterogeneity to investigate their genomic profile during their medical treatment.

KRAS mutations, with codons 12 and 13 mutations being the most frequent, are oncogenic drivers in lung cancer [27]. These mutations cause constitutive activation of the RAS signaling pathway, and further activate several downstream signaling effectors such as the canonical Raf-MEK-ERK, the PI3K-AKT-mTOR, RalGDS-RalA/B pathways and the TIAM1-RAC1 pathway [28]. The frequency of KRAS codon 61 mutation in our study was higher than the previously reported rate of TCGA cohorts (1\%) that included the large number of Caucasian patients, and similar to the frequency of East Asian (13\%) [29]. This discrepancy may partially attribute to the difference of ethnicity and age. Some studies have not found KRAS codon 61 mutations in the young patients with NSCLC $[30,31]$, and indicated that the incidence of codon 61 mutation was more frequent in the older patients with colorectal cancer [32]. Unlike G-C or G-T mutating more in patients with smoking history [33], all codon 61 mutations identified in our study were transversion mutation (A-T) and most of which were occurred in non-smokers. Overall, such cancers with codon 61 mutations may be caused by the accumulation of years other than environment tobacco exposure.

The prognostic value of TP53 mutations in EGFRmutated lung cancer is still a controversial issue. Some researchers suggested that TP53 mutations were associated with shorter survival in EGFR-mutated patients [34]. However, a couple of studies have failed to demonstrate this association between survival and TP53 mutations $[35,36]$, which was in line with our findings that harboring a TP53 mutation did not significantly affect PFS in EGFR-mutated patients. It might be speculated that TP53 mutation as a prognostic marker is unclear in lung cancer patients with EGFR mutation, and the TP53 status cannot be used to select treatment for EGFR-mutated patients until now. The influence of TP53 mutational status on EGFR-mutated patients with lung cancer is required to clarify with larger datasets.

Molecular targeted therapies have been developed to specifically block cancer growth [37]. Especially, EGFR TKIs are demonstrated to have ability to prolong survival time of EGFR-mutated patients with lung carcinoma [38, 39]. In concordance with these reports, targeted therapy is associated with better PFS and OS compared with conventional chemoradiotherapy in the current study.
Previous studies showed that molecular targeted therapy plus chemotherapy improved PFS compared with targeted therapy alone lung cancer patients [40, 41]. However, we did not get such data because of the relatively small sample size that treated with TKIs plus chemotherapy. For EGFR mutated elderly patients with poor performance status, EGFR TKIs, maybe a recommended option that could improve survival time compared with chemoradiotherapy alone.

\section{Conclusions}

In this study, aged patients with lung cancer showed some specific gene alterations, such as a relative high proportion KRAS codon 61 in the KRAS mutated patients, and the various mechanism of EGFR TKIs resistance. The use of NGS technology can help clinicians making personalized medicine strategies, especially distinguishing specific resistance mechanisms in each patient. Besides, these old patients in our cohort were benefit from the targeted treatment other than chemoradiotherapy alone. However, further studies are required to confirm these findings and a much larger sample size is needed to analyze the association of treatment selections with patient survival.

\section{Methods}

\section{Patients and samples}

We retrospectively analyzed 267 samples including 118 FFPE tissues and 149 plasma specimens from 258 lung carcinoma patients, collected in Central Hospital of Guangdong Nongken during December 2016 and December 2020. Based on the individual wishes of patients and their families, the patients were treated according to Chinese Medical Association guidelines for clinical diagnosis and treatment of lung cancer [42, 43] or the National Comprehensive Cancer Network guidelines [44, 45]. Written Informed consent was provided by all patients before testing. All the participants, samples and data involved in our study have been performed in accordance with the Declaration of Helsinki, and approved by the Ethics Committee of Central Hospital of Guangdong Nongken (No.2018001).

About $10 \mathrm{~mL}$ peripheral blood was collected from each patient into EDTA-containing tubes or cell-Free DNA $\mathrm{BCT}^{\circledR}$ tube (Streck Inc., Omaha, USA). Plasma was isolated using a double centrifugation protocol by centrifugation at $1600 \mathrm{~g}$ for $10 \mathrm{~min}$, followed by $16,000 \mathrm{~g}$ for $10 \mathrm{~min}$, and stored at $-80^{\circ} \mathrm{C}$ until subsequent analysis. Tumor tissues were fixed in $10 \%$ neutral buffered formalin overnight and were routinely embedded in paraffin, with conformation by the pathologists for diagnosis and tumor purity. 


\section{DNA extraction}

Genomic DNA was extracted from unstained $10-\mu \mathrm{m}$ thick FFPE sections with tumor content more than 10\% using the QIAamp DNA FFPE Tissue Kit (Qiagen, Hilden, Germany) following the manufacturer's instructions. Circulating cell-free DNA (cfDNA) was recovered from the plasma samples using the QIAamp Circulating Nucleic Acid kit (Qiagen). After extraction, DNA quality was evaluated by $1 \%$ agarose gel electrophoresis and the concentration of all samples was quantified using the Qubit dsDNA HS Assay kit (Thermo Fisher Scientific, Waltham, MA, USA) with a Qubit 3.0 Fluorometer.

\section{Next generation sequencing and data analysis}

A probe-based targeted NGS was used for library generating with an OncoAim ${ }^{\circledR}$ Lung cancer targeting gene detection kit (Singlera Genomics, Inc., Shanghai, China), of which the gene panel included all exons of 12 genes (ALK, BRAF, EGFR, ERBB2, FGFR1, KRAS, MET, NRAS, $P I K 3 C A, R E T, R O S 1, T P 53)$ involved in tumorigenesis, and potential gene rearrangement/fusion of $A L K$, ROS1 and RET. According to the kit protocol, the input of FFPE DNA and cfDNA for library preparation was $50 \mathrm{ng}$ and 30 ng, respectively. Accordingly, FFPE DNA were sheared to about $250 \mathrm{bp}$ with restriction enzyme before library construction. The cfDNA libraries were constructed with 12 bp unique molecular identifier (UMI) sequences to distinguish PCR-duplicated fragments. After the end repair, A-tailing, and adapter ligation, target capture with probes supplied in the kit was performed according to manufacturer's specifications. The library product was sequenced using $150 \mathrm{bp}$ paired-end runs on the NextSeq 500 (Illumina, Inc., San Diego, CA, USA), with an average sequencing depth of FFPE library and cfDNA was $1000 \mathrm{X}$ and 20,000X, separately.

Sequencing data were processed following the guideline of the OncoAim ${ }^{\circledR}$ kit (Singlera), which was designed to simultaneously detect single nucleotide variations (SNV), short insertions and deletions (InDels), copy number variations (CNV) and gene rearrangements. Briefly, sequencing reads were quality-filtered with FastQC (version 0.9.5, Babraham Bioinformatics, Cambridge, UK), and assembled and aligned against the reference genome hg19/GRCh37 by the Burrow-Wheeler Aligner algorithm (https://github.com/lh3/bwa; version 0.7.12-r1039; Dec 2015). Unique reads derived from GATK were used for variant calling. Insertions and deletions in sequence alignment files were left-aligned using Freebayes (https://github.com/ekg/freebayes). For the NGS data sequenced from cfDNA samples, the UMI was used as a variants filter. The minimum confidence threshold for variant and insertion/deletion (indel) calling was set to $0.001(0.1 \%)$ for cfDNA samples and $0.02 \%$ (2\%) for FFPE samples.

\section{Statistical analysis}

Statistical analysis was performed using $\mathrm{R}$ version 4.0.4 (R Development Core Team, 2019) and SPSS 22.0 software (SPSS, Chicago, USA). The chi-square test or Fisher's test was used to analyze the association of mutational status detected for the first sampling in our study with clinical features. The Kaplan-Meier method with a logrank test was used to estimate the median progression free survival (PFS) and median overall survival (OS). $P$ value $<0.05$ was considered statistically significant.

\section{Abbreviations}

CT: Computed tomography; PET: Positron emission tomography; NGS: Next generation sequencing; FFPE: Formalin-fixed paraffin-embedded; cfDNA: Circulating cell-free DNA; SNV: Single nucleotide variations; InDels: Short insertions and deletions; CNV: Copy number variations; PFS: Progression free survival; OS: Overall survival; TKIs: Tyrosine kinase inhibitors; ADC: Adenocarcinoma; SCC: Squamous cell carcinoma; ASC: Adenosquamous carcinoma; 19Del: Exon 19 deletions; ARMS: Amplification Refractory Mutation System; PR: Partial responses; PD: Progression disease; SD: Stable disease; BSC: Best supportive care; MTT: Molecular targeted therapy; CRT: Chemoradiotherapy.

\section{Supplementary Information}

The online version contains supplementary material available at https://doi. org/10.1186/s12864-021-08289-4.

Additional file 1: Table S1-S4.

Additional file 2: Figure S1.

\section{Acknowledgements}

Not applicable.

\section{Authors' contributions}

SXC and AZF: design. YL and YFC: acquisition of data. WL: validation. AZF, SQH and SLZ: analysis and interpretation of data. SXC, ZZZ and TMX: writing the manuscript. YGC: Funding Acquisition. All authors read and approved the final manuscript.

\section{Funding}

This work was supported by the Project of Science and Technology Plan of Zhanjiang City (2017A304)

\section{Availability of data and materials}

All data generated or analyzed during this study are included within the article.

\section{Declarations}

Ethics approval and consent to participate

All the participants, samples, data and experimental protocols involved in our study have been performed in accordance with the Declaration of Helsinki, and approved by the Ethics Committee of Central Hospital of Guangdong Nongken (No.2018001). Written Informed consent was provided by all patients.

\section{Consent for publication}

Not applicable. 


\section{Competing interests}

The authors declare that they have no competing interests.

\section{Author details}

${ }^{1}$ Medical Oncology Department V, Guangdong Nongken Central Hospital, Zhanjiang 524002, China. ${ }^{2}$ Gynecology Department, Affiliated Hospital of Guangdong Medical University, Zhanjiang 524001, China. ${ }^{3}$ Singlera Genomics Inc., Shanghai 201318, China.

Received: 22 June 2021 Accepted: 28 December 2021

Published online: 15 January 2022

\section{References}

1. Siegel RL, Miller KD, Fuchs HE, Jemal A. Cancer Statistics, 2021. CA Cancer J Clin. 2021;71(1):7-33.

2. Galvez-Nino M, Ruiz R, Pinto JA, Roque K, Mantilla R, Raez LE, et al. Lung Cancer in the young. Lung. 2020;198(1):195-200.

3. Barta JA, Zinner RG, Unger M. Lung Cancer in the older patient. Clin Geriatr Med. 2017;33(4):563-77.

4. Ganti AK, deShazo M, Weir AB 3rd, Hurria A. Treatment of non-small cell lung cancer in the older patient. J Natl Compr Cancer Netw. 2012:10(2):230-9.

5. Presley CJ, Gross CP, Lilenbaum RC. Optimizing Treatment Risk and Benefit for Elderly Patients With Advanced Non-Small-Cell Lung Cancer: The Right Treatment for the Right Patient. J Clin Oncol. 2016;34(13):1438-42.

6. Dutkowska AE, Antczak A. Comorbidities in lung cancer. Pneumonol Alergol Pol. 2016;84(3):186-92.

7. Mayekar MK, Bivona TG. Current landscape of targeted therapy in lung Cancer. Clin Pharmacol Ther. 2017;102(5):757-64.

8. Lindeman NI, Cagle PT, Aisner DL, Arcila ME, Beasley MB, Bernicker EH, et al. Updated molecular testing guideline for the selection of lung Cancer patients for treatment with targeted tyrosine kinase inhibitors: guideline from the College of American Pathologists, the International Association for the Study of Lung Cancer, and the Association for Molecular Pathology. J Thorac Oncol. 2018;13(3):323-58.

9. Reungwetwattana T, Weroha SJ, Molina JR. Oncogenic pathways, molecularly targeted therapies, and highlighted clinical trials in non-small-cell lung cancer (NSCLC). Clin Lung Cancer. 2012;13(4):252-66.

10. Genova C, Rossi G, Tagliamento M, Rijavec E, Biello F, Cerbone L, et al. Targeted therapy of oncogenic-driven advanced non-small cell lung cancer: recent advances and new perspectives. Expert rev. Respir Med. 2020:14(4):367-83.

11. Aberle DR, Berg CD, Black WC, Church TR, Fagerstrom RM, Galen B, et al. The National Lung Screening Trial: overview and study design. Radiology. 2011;258(1):243-53.

12. Shi Y, Au JS, Thongprasert S, Srinivasan S, Tsai CM, Khoa MT, et al. A prospective, molecular epidemiology study of EGFR mutations in Asian patients with advanced non-small-cell lung cancer of adenocarcinoma histology (PIONEER). J Thorac Oncol. 2014;9(2):154-62.

13. Bareschino MA, Schettino C, Rossi A, Maione P, Sacco PC, Zeppa R, et al. Treatment of advanced non small cell lung cancer. J Thorac Dis. 2011;3(2):122-33.

14. Colombino M, Paliogiannis $P$, Cossu A, Santeufemia DA, Sini MC, Casula M, et al. EGFR, KRAS, BRAF, ALK, and CMET genetic alterations in 1440 Sardinian patients with lung adenocarcinoma. BMC Pulm Med. 2019;19(1):209.

15. Halvorsen AR, Silwal-Pandit L, Meza-Zepeda LA, Vodak D, Vu P, Sagerup $C$, et al. TP53 mutation Spectrum in smokers and never smoking lung Cancer patients. Front Genet. 2016;7:85.

16. Zheng $\mathrm{HC}$. The molecular mechanisms of chemoresistance in cancers. Oncotarget. 2017;8(35):59950-64.

17. Yang CY, Yang JC, Yang PC. Precision Management of Advanced nonSmall Cell Lung Cancer. Annu Rev Med. 2020;71:117-36.

18. Iuchi T, Shingyoji M, Sakaida T, Hatano K, Nagano O, Itakura M, et al. Phase II trial of gefitinib alone without radiation therapy for Japanese patients with brain metastases from EGFR-mutant lung adenocarcinoma. Lung Cancer. 2013:82(2):282-7.

19. Singh $M$, Jadhav HR. Targeting non-small cell lung cancer with small-molecule EGFR tyrosine kinase inhibitors. Drug Discov Today. 2018;23(3):745-53.
20. Ke EE, Zhou Q, Zhang QY, Su J, Chen ZH, Zhang XC, et al. A higher proportion of the EGFR T790M mutation may contribute to the better survival of patients with exon 19 deletions compared with those with L858R. J Thorac Oncol. 2017;12(9):1368-75.

21. Chung KP, Wu SG, Wu JY, Yang JC, Yu CJ, Wei PF, et al. Clinical outcomes in non-small cell lung cancers harboring different exon 19 deletions in EGFR. Clin Cancer Res. 2012;18(12):3470-7.

22. Zhao C, Jiang T, Li J, Wang Y, Su C, Chen X, et al. The impact of EGFR exon 19 deletion subtypes on clinical outcomes in non-small cell lung cancer. Transl lung. Cancer Res. 2020;9(4):1149-58.

23. Westover D, Zugazagoitia J, Cho BC, Lovly CM, Paz-Ares L. Mechanisms of acquired resistance to first- and second-generation EGFR tyrosine kinase inhibitors. Ann Oncol. 2018:29(Suppl 1):i10-9.

24. Kobayashi S, Boggon TJ, Dayaram T, Jänne PA, Kocher O, Meyerson M, et al. EGFR mutation and resistance of non-small-cell lung cancer to gefitinib. N Engl J Med. 2005;352(8):786-92.

25. Yun CH, Mengwasser KE, Toms AV, Woo MS, Greulich H, Wong KK, et al. The T790M mutation in EGFR kinase causes drug resistance by increasing the affinity for ATP. Proc Natl Acad Sci U S A. 2008;105(6):2070-5.

26. Oxnard GR, Hu Y, Mileham KF, Husain H, Costa DB, Tracy P, et al. Assessment of resistance mechanisms and clinical implications in patients with EGFR T790M-positive lung Cancer and acquired resistance to Osimertinib. JAMA. Oncol. 2018;4(11):1527-34.

27. Lindsay CR, Jamal-Hanjani M, Forster M, Blackhall F. KRAS: reasons for optimism in lung cancer. Eur J Cancer. 2018;99:20-7.

28. Román M, Baraibar I, López I, Nadal E, Rolfo C, Vicent S, et al. KRAS oncogene in non-small cell lung cancer: clinical perspectives on the treatment of an old target. Mol Cancer. 2018;17(1):33

29. Liu L, Liu J, Shao D, Deng Q, Tang H, Liu Z, et al. Comprehensive genomic profiling of lung cancer using a validated panel to explore therapeutic targets in east Asian patients. Cancer Sci. 2017;108(12):2487-94.

30. Yang B, Li J, Li F, Zhou H, Shi W, Shi H, et al. Comprehensive analysis of age-related somatic mutation profiles in Chinese young lung adenocarcinoma patients. Cancer Med. 2019;8(4):1350-8.

31. Chen Z, Teng X, Zhang J, Huang K, Shen Q, Cao H, et al. Molecular features of lung adenocarcinoma in young patients. BMC Cancer. 2019;19(1):777.

32. Serebriiskii IG, Connelly C, Frampton G, Newberg J, Cooke M, Miller V, et al. Comprehensive characterization of RAS mutations in colon and rectal cancers in old and young patients. Nat Commun. 2019;10(1):3722.

33. Riely GJ, Kris MG, Rosenbaum D, Marks J, Li A, Chitale DA, et al. Frequency and distinctive spectrum of KRAS mutations in never smokers with lung adenocarcinoma. Clin Cancer Res. 2008:14(18):5731-4.

34. Hou H, Qin K, Liang Y, Zhang C, Liu D, Jiang H, et al. Concurrent TP53 mutations predict poor outcomes of EGFR-TKI treatments in Chinese patients with advanced NSCLC. Cancer Manag Res. 2019;11:5665-75.

35. Shepherd FA, Lacas B, Le Teuff G, Hainaut $P$, Janne PA, Pignon JP, et al. Pooled analysis of the prognostic and predictive effects of TP53 Comutation status combined with KRAS or EGFR mutation in early-stage resected non-small-cell lung Cancer in four trials of adjuvant chemotherapy. J Clin Oncol. 2017;35(18):2018-27.

36. Labbe C, Cabanero M, Korpanty GJ, Tomasini P, Doherty MK, Mascaux C, et al. Prognostic and predictive effects of TP53 co-mutation in patients with EGFR-mutated non-small cell lung cancer (NSCLC). Lung Cancer. 2017:111:23-9.

37. Lee YT, Tan YJ, Oon CE. Molecular targeted therapy: treating cancer with specificity. Eur J Pharmacol. 2018;834:188-96.

38. Rosell R, Carcereny E, Gervais R, Vergnenegre A, Massuti B, Felip E, et al. Erlotinib versus standard chemotherapy as first-line treatment for European patients with advanced EGFR mutation-positive non-small-cell lung cancer (EURTAC): a multicentre, open-label, randomised phase 3 trial. Lancet Oncol. 2012;13(3):239-46.

39. Kuan FC, Kuo LT, Chen MC, Yang CT, Shi CS, Teng D, et al. Overall surviva benefits of first-line EGFR tyrosine kinase inhibitors in EGFR-mutated non-small-cell lung cancers: a systematic review and meta-analysis. $\mathrm{Br}$ J Cancer. 2015;113(10):1519-28.

40. Cheng Y, Murakami H, Yang PC, He J, Nakagawa K, Kang JH, et al. Randomized phase II trial of Gefitinib with and without Pemetrexed as first-line therapy in patients with advanced nonsquamous non-small-cell lung Cancer with activating epidermal growth factor receptor mutations. Clin Oncol. 2016;34(27):3258-66. 
41. Hosomi Y, Morita S, Sugawara S, Kato T, Fukuhara T, Gemma A, et al. Gefitinib alone versus Gefitinib plus chemotherapy for non-small-cell lung Cancer with mutated epidermal growth factor receptor: NEJ009 study. J Clin Oncol. 2020;38(2):115-23.

42. Chinese Medical Association, Oncology Society of Chinese Medical Association, Chinese Medical Association Publishing House. Chinese Medical Association guidelines for clinical diagnosis and treatment of lung cancer (Edition 2018). Chin J Oncol. 2018;40(12):935-64.

43. Chinese Medical Association. Chinese Medical Association Guidelines for Clinical Diagnosis and Treatment of Lung Cancer (2019 Edition). Chin J Oncol. 2020;42:(4):257-87.

44. Ettinger DS, Aisner DL, Wood DE, Akerley W, Bauman J, Chang JY, et al. NCCN guidelines insights: non-small cell lung Cancer, version 5.2018. J Natl Compr Cancer Netw. 2018;16(7):807-21.

45. Ettinger DS, Wood DE, Aggarwal C, Aisner DL, Akerley W, Bauman JR, et al. NCCN guidelines insights: non-small cell lung Cancer, version 1.2020. J Natl Compr Cancer Netw. 2019;17(12):1464-72.

\section{Publisher's Note}

Springer Nature remains neutral with regard to jurisdictional claims in published maps and institutional affiliations.

- fast, convenient online submission

- thorough peer review by experienced researchers in your field

- rapid publication on acceptance

- support for research data, including large and complex data types

- gold Open Access which fosters wider collaboration and increased citations

- maximum visibility for your research: over $100 \mathrm{M}$ website views per year

At BMC, research is always in progress.

Learn more biomedcentral.com/submissions 